\title{
Endothelial Dysfunction in Diabetic Patients with Abnormal Myocardial Perfusion in the Absence of Epicardial Obstructive Coronary Artery Disease
}

Roxana Djaberi ${ }^{1}$, Jos op 't Roodt ${ }^{2}$, Joanne D. Schuijf ${ }^{1}$, Ton J. Rabelink ${ }^{2}$, Eelco J. de Koning ${ }^{2}$, Alberto M. Pereira ${ }^{3}$, Marcel P. Stokkel ${ }^{4}$, Jan W. Smit ${ }^{3}$, Jeroen J. Bax ${ }^{1}$, and J. Wouter Jukema ${ }^{1,5}$

${ }^{I}$ Department of Cardiology, Leiden University Medical Center, Leiden, The Netherlands; ${ }^{2}$ Department of Nephrology, Leiden University Medical Center, Leiden, The Netherlands; ${ }^{3}$ Department of Endocrinology, Leiden University Medical Center, Leiden, The Netherlands; ${ }^{4}$ Department of Nuclear Medicine, Leiden University Medical Center, Leiden, The Netherlands; and ${ }^{5}$ Einthoven Laboratory of Experimental Vascular Medicine, Leiden, The Netherlands

In patients with diabetes mellitus, myocardial perfusion defects are often observed in the absence of obstructive epicardial coronary artery disease (CAD), thereby presenting a diagnostic problem. We hypothesized that these perfusion abnormalities may be explained by endothelial dysfunction or occult coronary atherosclerosis. Methods: Prospectively, 130 asymptomatic patients with diabetes mellitus underwent cardiovascular screening by coronary artery calcium (CAC) scoring, multislice CT coronary angiography, and myocardial perfusion imaging by SPECT. Multislice CT images were evaluated for the presence of obstructive epicardial CAD ( $\geq 50 \%$ luminal narrowing). To quantify abnormal myocardial perfusion on SPECT images, we determined the summed stress score for each patient. The presence of abnormal myocardial perfusion was defined as a summed stress score of 3 or more. In addition, flow-mediated dilatation of the brachial artery, a marker of endothelial function, was determined using ultrasonography. Results: In 35 patients (27\%), obstructive epicardial CAD was observed on multislice $\mathrm{CT}$, and these patients were excluded from further analysis. In the remaining 95 patients, abnormal myocardial perfusion was observed in 30 (32\%) of patients. Flow-mediated dilatation was significantly lower in patients with abnormal myocardial perfusion $(3.6 \% \pm 2.4 \%)$ than in those with normal myocardial perfusion $(6.4 \% \pm 2.6 \%)(P<0.001)$. Importantly, flow-mediated dilatation remained a significant predictor of the extent of abnormal myocardial perfusion after correction for cardiovascular risk factors and CAC score $(P<0.001)$. In contrast, no association was observed between nonobstructive plaque burden as reflected by CAC scores and extent of abnormal myocardial perfusion. Conclusion: In patients with diabetes mellitus, myocardial perfusion abnormalities in the absence of obstructive epicardial CAD are associated with endothelial dysfunction.

Received Apr. 15, 2009; revision accepted Aug. 20, 2009.

For correspondence or reprints contact: Jeroen J. Bax, Department of Cardiology, Leiden University Medical Center, Postbus 9600, 2300 RC,

Leiden, The Netherlands.

E-mail: J.J.Bax@lumc.nl

COPYRIGHT $\odot 2009$ by the Society of Nuclear Medicine, Inc.
Key Words: diabetes mellitus; endothelium; myocardial perfusion; coronary artery disease

J Nucl Med 2009; 50:1980-1986

DOI: 10.2967/jnumed.109.065193

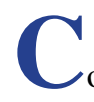
oronary artery disease (CAD) constitutes a major cause of morbidity and mortality in patients with diabetes mellitus. In addition, because of diabetic neuropathy, myocardial ischemia and infarction may be prevalent in the absence of typical anginal symptoms. Noninvasive assessment of CAD in asymptomatic diabetic patients at higher risk has therefore been previously suggested by the guidelines of the American Diabetes Association and the American Heart Association (1). Those guidelines propose testing for the presence of myocardial ischemia by SPECT as a possible option for early detection and treatment of asymptomatic obstructive CAD. If myocardial perfusion abnormalities are identified, coronary angiography is considered to confirm the presence of clinically relevant obstructive CAD possibly requiring intervention. However, previous studies have shown a discrepancy between the presence of myocardial perfusion defects and obstructive CAD in diabetic patients. In approximately $20 \%-40 \%$ of diabetic patients, myocardial perfusion defects could not be clarified by obstruction of the epicardial coronary arteries $(2,3)$. Also, in the general population, a discrepancy between the presence of myocardial perfusion defects and obstructive epicardial CAD has been observed (4-6). Both endothelial dysfunction and occult coronary atherosclerosis were shown to contribute to this condition (4-6). However, the potential mechanisms underlying myocardial perfusion abnormalities not attributable to obstruction of flow in the epicardial coronary arteries have not yet been studied in asymptomatic diabetic patients. 
Ultrasonographic measurement of flow-mediated dilatation (FMD) of the brachial artery provides a noninvasive estimation of systemic endothelial function (7). The observed brachial artery dilatation has been shown to be closely related to coronary endothelial function and vasoreactivity $(8,9)$. The reproducibility of assessments of brachial artery diameter has improved with the development of the wall-track system, rendering it suitable for cohort studies (10).

The current study was designed to prospectively evaluate the relationship between myocardial perfusion as assessed by SPECT and endothelial function as assessed by FMD in asymptomatic diabetic patients without epicardial obstructive CAD (as evaluated noninvasively by multislice CT coronary angiography). In addition, the association between nonobstructive plaque burden as reflected by coronary artery calcium (CAC) scores and myocardial perfusion was studied.

\section{MATERIALS AND METHODS}

\section{Patients and Design}

Prospectively, 130 asymptomatic diabetic patients were screened for cardiovascular disease. A structured interview, physical examination, and laboratory analysis were performed. Cardiovascular risk factors were assessed according to the following criteria. A family history of CAD was considered positive if CAD was present in any first-degree family member. Hypertension was defined as blood pressure greater than $140 / 90 \mathrm{~mm} \mathrm{Hg}$ or treatment with antihypertensive medication. Hypercholesterolemia was defined as a total cholesterol level greater than $5.0 \mathrm{mmol} / \mathrm{L}$ or the use of lipid-lowering medication.

Noninvasive multislice CT, including CAC scoring and coronary angiography, and myocardial perfusion imaging by SPECT were performed as part of the clinical work-up of all patients. Concurrent measurement of brachial FMD was performed in a study setting approved by the institutional review committee of the Leiden University Medical Center.

Primarily, patients not eligible for multislice CT coronary angiography because of arrhythmia or contraindications to the use of iodinated contrast medium were excluded. Subsequently, those with obstructive epicardial CAD on multislice CT angiography were excluded from further analyses. The relationship between the presence of myocardial perfusion defects and FMD was thereby assessed in the remainder of patients free of obstructive epicardial CAD.

\section{Multislice CT Data Acquisition}

Imaging was performed with a 64-slice multislice CT scanner (Toshiba Medical Systems). In patients with a heart rate of at least 65 beats per minute, oral $\beta$-blocking medication (metoprolol, $50 \mathrm{mg}$ or $100 \mathrm{mg}$ ), if tolerated, was provided $1 \mathrm{~h}$ preceding the scan. A nonenhanced prospective electrocardiographically gated scan, triggered at $75 \%$ of the R-R interval with $4 \times 3.0$-mm collimation was first obtained to measure the CAC score and to determine the start and end positions of the helical scan.

Subsequently, multislice CT angiography was performed using the following parameters: collimation, $64 \times 0.5 \mathrm{~mm}$; tube rotation time, 400,450 , or $500 \mathrm{~ms}$, depending on the heart rate; tube current, 300 or $350 \mathrm{~mA}$; and tube voltage, $120 \mathrm{kV}$. Nonionic contrast material was administered in the antecubital vein $(90-100 \mathrm{~mL}$, depending on the total scan time) at a flow rate of $5 \mathrm{~mL} / \mathrm{s}$, followed by $50 \mathrm{~mL}$ of saline solution flush. Timing of the scan was determined by automated bolus tracking in the aortic root. Data were acquired with simultaneous electrocardiography registration during a single breath-hold of approximately $10 \mathrm{~s}$. Images were reconstructed in the cardiac phase that showed the fewest motion artifacts and were transferred to a remote workstation (Vitrea 2; Vital Images) for postprocessing.

\section{Multislice CT Data Analysis}

CAC Score. The CAC score was assessed using dedicated software (Vitrea 2). CAC was defined as a dense area in the coronary artery exceeding the threshold of 130 Hounsfield units. For each patient, the total Agatston score was determined.

Epicardial Obstructive CAD. Two experienced observers who were unaware of patient characteristics interpreted all multislice CT coronary angiograms. Discrepancies in interpretation were instantly resolved by consensus. Epicardial coronary arteries were examined by scrolling through axial images, followed by visual assessment of curved multiplanar reconstructions in at least 2 orthogonal planes. The presence of epicardial obstructive CAD (defined as luminal narrowing $\geq 50 \%$ in at least 1 vessel) was evaluated for each patient.

\section{ECG-Gated SPECT Data Acquisition}

During a 2-d stress and rest protocol, myocardial perfusion imaging was performed using ECG-gated SPECT with ${ }^{99 m} \mathrm{Tc}-$ sestamibi. Patients were instructed to abstain from caffeinecontaining products for $24 \mathrm{~h}$ preceding the stress test. Stress was induced using an intravenous infusion of adenosine at a rate of $140 \mu \mathrm{g} / \mathrm{kg}$ of body weight per minute for $6 \mathrm{~min}$, accompanied by simultaneous handgrip exercise. After completion of the third minute, ${ }^{99 \mathrm{~m}} \mathrm{Tc}$-sestamibi (500 $\mathrm{MBq}$ ) was injected intravenously. Blood pressure and a 12-lead electrocardiograph were recorded throughout the adenosine infusion. Imaging using a triple-head SPECT $\gamma$-camera (GCA 9300/HG; Toshiba Corp.) equipped with low-energy high-resolution collimators commenced 120 min after radiopharmaceutical injection. Images were acquired in accordance with American Society of Nuclear Cardiology guidelines, using a circular $360^{\circ}$ orbit, 60 projections, and $40 \mathrm{~s}$ per projection. Attenuation correction was not applied. Images were processed in the usual manner, and short-axis, horizontal long-axis, and vertical long-axis views were reconstructed. Patient motion was evaluated through examination of the raw cine images.

\section{SPECT Data Analysis}

Short-axis slices were displayed in polar map format, adjusted for peak myocardial activity of $100 \%$. Additional reconstruction yielded standard long- and short-axis projections perpendicular to the heart axis. All views were used for semiquantitative interpretation. As proposed by the American Society of Nuclear Cardiology guidelines, the myocardium was divided into 17 segments (11). Tracer uptake in each segment was evaluated by 2 observers in consensus, by use of a 5-point scoring system $(0$, normal reduction of tracer uptake; 1 , mild reduction of tracer uptake; 2 , moderate reduction of tracer uptake; 3 , severe reduction of tracer uptake; and 4, apparent absence of tracer uptake). The total segmental score during stress and rest was used to determine the summed stress score (SSS) and summed rest score for each patient. Abnormal myocardial perfusion was defined as an SSS of at least 3. Patients with an SSS of 3-7 were classified as having 
moderately abnormal myocardial perfusion, and those with an SSS of at least 8 were classified as having severely abnormal myocardial perfusion. The location of myocardial perfusion abnormalities was classified as apical, anterior, inferior, or lateral (11). Finally, regional wall motion on gated SPECT images was analyzed to differentiate between true perfusion abnormalities and attenuation artifacts.

\section{FMD}

The brachial artery diameter was evaluated using a wall-track system (Scanner 200; Pie Medical), which consisted of an ultrasound imager with a 7.5-MHz linear array transducer connected to a data acquisition system and a personal computer. The principles of this system have been previously described in more detail (12). All measurements were performed by the same experienced sonographer, who was unaware of the clinical characteristics of the patients. On the basis of a previous study of 20 healthy middleaged volunteers, the intraobserver coefficients of variation for FMD assessment by the same sonographer were previously determined to be $0.8 \%$ for the baseline diameter of the brachial artery, $2.0 \%$ for the maximal diameter, $24.2 \%$ for the change in diameter, and $24.4 \%$ for the calculated FMD. The mean FMD in this group of healthy volunteers was $8.4 \% \pm 2.1 \%$.

In the current study, patients were asked to abstain from medication use, caffeine-containing substances, and smoking for $24 \mathrm{~h}$ before the tests. All measurements were performed on fasting patients, in the morning, in a silent, temperature-controlled clinical research laboratory. Patients had a 15-min rest in a comfortable supine position and remained in that position throughout the examination. The patients' right arm was placed in extension in the elbow, with the hand in supination, thus eliminating longitudinal movements of the arm. The wrist and elbow were supported by cushions to minimize lateral movements. The heart rate was continuously monitored by a 3-lead electrocardiogram. The brachial artery was visualized distal to the elbow. First, 3 diastolic measurements were averaged to provide a baseline diameter. Then, ischemia was applied to the forearm distal to the location of the transducer by inflation of a blood pressure cuff for $5 \mathrm{~min}$ at a pressure of $200 \mathrm{~mm} \mathrm{Hg}$. After cuff deflation, ultrasonography continued for $5 \mathrm{~min}$ with measurements at 30 -s intervals. The widest lumen diameter was used as maximal vasodilation. FMD was expressed as percentage change relative to the baseline diameter.

\section{Statistical Analysis}

Primarily, patients with obstructive epicardial CAD $(\geq 50 \%$ luminal narrowing) on multislice CT angiography were identified and excluded from all further analysis.

Continuous variables were expressed as mean $\pm \mathrm{SD}$. Categoric variables were expressed as number, followed by percentage in parentheses.

To investigate the relationship between FMD and myocardial perfusion, we first stratified patients as having either normal myocardial perfusion (SSS $<3$ ) or abnormal myocardial perfusion (SSS $\geq 3$ ) on SPECT. Average FMD and SD were calculated for both groups, and the independent $t$ test was applied to evaluate the difference in mean FMD.

To determine the relationship between cardiovascular risk factors and degree of myocardial perfusion abnormalities, a univariate linear regression analysis of baseline characteristics was performed to identify the potential predictors of the number of SSS. Thereafter, multivariate linear regression analysis with back- ward elimination was performed to identify the independent predictors of the number of SSS.

Finally, the relationship between FMD and prevalence of abnormal myocardial perfusion, as well as between the degree of nonobstructive atherosclerosis and abnormal myocardial perfusion, were evaluated. For this purpose, patients were first categorized according to FMD quartiles, and the prevalence of abnormal myocardial perfusion was determined for each FMD quartile. Subsequently, patients were stratified as having a CAC score of 0 , $1-10,11-100$, or more than 100 , and the prevalence of abnormal myocardial perfusion was assessed per CAC category. The influence of decreasing FMD (per quartile) and that of increasing CAC scores (per category) on the prevalence of abnormal myocardial perfusion was investigated.

\section{RESULTS}

\section{Patient Characteristics}

In the total population, multislice CT coronary angiography revealed obstructive epicardial $\mathrm{CAD}(\geq 50 \%$ luminal narrowing) in 35 patients $(27 \%)$, and these patients were therefore excluded. The characteristics of the remaining 95 patients $(73 \%)$ included in the analysis are provided in Table 1 . Briefly, the mean age of this population was $48 \pm$ $12 \mathrm{y}$, and 43 patients (46\%) were male.

\section{Baseline Imaging Findings}

Assessment of Myocardial Perfusion by SPECT. The mean SSS was $2.0 \pm 3.0$ (range, $0-13$ ) in patients included in the analysis. In this asymptomatic diabetic population without obstructive epicardial CAD, myocardial perfusion

\begin{tabular}{|c|c|}
\hline Characteristic & Value \\
\hline Age (y) & $48 \pm 12$ \\
\hline Male sex & $43(46 \%)$ \\
\hline Diabetes mellitus duration (y) & $15 \pm 13$ \\
\hline Body mass index $\left(\mathrm{kg} / \mathrm{m}^{2}\right)$ & $28 \pm 6$ \\
\hline Hemoglobin A1c (mmol/L) & $8.0 \pm 1.6$ \\
\hline Smoking & $23(25 \%)$ \\
\hline Family history of CAD & $42(45 \%)$ \\
\hline Hypercholesterolemia & $62(66 \%)$ \\
\hline Hypertension & $47(50 \%)$ \\
\hline Type 1/type 2 diabetes mellitus & $45 / 50$ \\
\hline \multicolumn{2}{|l|}{ Diabetes treatment } \\
\hline Diet only & $5(5 \%)$ \\
\hline Oral agent & $14(15 \%)$ \\
\hline Oral agent and insulin & $21(22 \%)$ \\
\hline Insulin & $54(57 \%)$ \\
\hline \multicolumn{2}{|l|}{ Cardiovascular medication use } \\
\hline Statin & $41(43 \%)$ \\
\hline Aspirin & $14(15 \%)$ \\
\hline Angiotensin-converting enzyme inhibitor & $15(16 \%)$ \\
\hline$\beta$-blocker & $9(9 \%)$ \\
\hline Angiotensin II antagonist & $12(13 \%)$ \\
\hline Calcium channel blocker & $7(7 \%)$ \\
\hline Diureticum & $10(11 \%)$ \\
\hline
\end{tabular}


abnormalities (SSS $\geq 3$ ) were observed in 30 patients $(32 \%)$. Within this group, moderately abnormal myocardial perfusion (SSS 3-7) was revealed in 25 patients $(83 \%)$, whereas 5 patients $(17 \%)$ were shown to have severely abnormal myocardial perfusion (SSS $\geq 8$ ). Furthermore, observed myocardial perfusion abnormalities were reversible in 14 patients $(47 \%)$, partially reversible in $6(20 \%)$, and fixed in $10(33 \%)$. Of note, fixed myocardial perfusion abnormalities comprised mainly a light or moderate decrease in tracer uptake $(n=8)$. Only 2 patients with a fixed abnormality were shown to have a severe decrease in tracer uptake. In total, 6 patients showed abnormal perfusion apically: 16 patients in the anterior wall of the myocardium, 15 in the inferior wall, and 8 in the lateral wall. Overall, 16 patients showed a myocardial perfusion abnormality in a single myocardial wall, 13 patients in 2 walls, and 1 patient in 3 walls.

Assessment of Endothelial Function by FMD. Mean FMD was $6.0 \% \pm 3.0 \%$, and FMD ranged from $0.0 \%$ to $13.3 \%$. The median FMD was calculated to be $5.4 \%$ (lower quartile, $3.4 \%$; upper quartile, $7.3 \%$ ).

Assessment of CAC Score by Multislice CT. The average CAC score was $60 \pm 170$. Most patients $(n=53,55 \%)$ had no coronary calcium. The CAC score was found to be in the range of $1-10$ in 12 patients (13\%), 11-100 in 15 patients (16\%), and more than 100 in 15 patients $(16 \%)$.

\section{Relationship Between FMD and Abnormal Myocardial Perfusion}

In diabetic patients with normal myocardial perfusion, the mean FMD was significantly higher $(6.4 \% \pm 2.6 \%)$ than in patients with abnormal myocardial perfusion $(3.6 \% \pm 2.4 \%)$ $(P<0.001)$ (Fig. 1).

The prevalence of patients with abnormal myocardial perfusion per FMD quartile or CAC score category is provided in Figure 2A. Interestingly, the prevalence of myo-

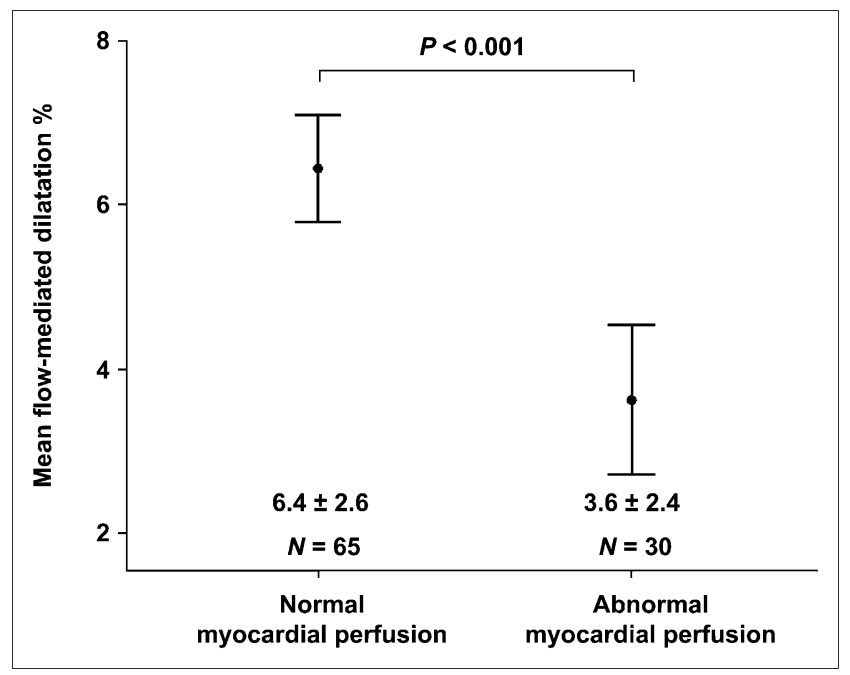

FIGURE 1. FMD in diabetic patients with normal and abnormal myocardial perfusion. Mean FMD was significantly lower in patients with abnormal myocardial perfusion. cardial perfusion abnormalities increased gradually from $9 \%$ to $61 \%$ with decreasing FMD quartiles.

In contrast, no trend was evident between the prevalence of abnormal myocardial perfusion and increasing CAC score categories (Fig. 2B). Although $34 \%$ of patients without coronary calcium showed abnormal myocardial perfusion, a similar prevalence $(40 \%)$ was observed in patients with extensive coronary calcium $(\mathrm{CAC}>100)$.

\section{Predictors of the Extent of Abnormal Myocardial Perfusion}

As illustrated in Table 2, FMD was the only significant predictor of the extent of abnormal myocardial perfusion in a univariate model $(P<0.001)$. Importantly, after correction for other cardiovascular risk factors in a multivariate model with backward elimination, FMD remained a significant predictor of the extent of abnormal myocardial perfusion $(P<0.001)$ (Table 2). Hemoglobin A1c and hypercholesterolemia were also shown to be independent predictors of the extent of abnormal myocardial perfusion.

\section{DISCUSSION}

The current prospective study revealed that $32 \%$ of asymptomatic patients with diabetes mellitus had abnormal myocardial perfusion despite the absence of obstructive epicardial CAD. Assessment of brachial FMD showed less vasoreactivity in these patients than in those with normal myocardial perfusion. Importantly, after correction for risk factors and CAC scores, endothelial function remained a significant predictor of abnormal myocardial perfusion. Further analysis showed the prevalence of myocardial perfusion abnormalities to increase per decreasing FMD quartile, whereas no such trend was observed for occult atherosclerotic plaque burden as determined by CAC scoring.

\section{Assessment of Myocardial Perfusion by SPECT}

In the general population of patients with known or suspected CAD, myocardial perfusion imaging by SPECT has been shown to successfully identify obstructive CAD and predict future coronary events $(13,14)$. The prognostic value of myocardial perfusion has also been confirmed in diabetic patients with suspected CAD, as well as in asymptomatic diabetic patients (15-17). Based on these observations in combination with the noninvasive nature of MPI by SPECT, the American Diabetes Association and the American Heart Association have proposed this technique as a potential screening tool for identification of asymptomatic diabetic patients with obstructive CAD (1). However, previous studies in diabetic patients imply that a significant proportion of observed perfusion abnormalities is not attributable to obstruction of blood flow in the epicardial coronary arteries $(2,3)$. In the general population, a similar discrepancy has been observed regarding presentation with anginal symptoms and manifested ischemia in the absence of obstructive epicardial CAD (4-6). Mechanisms underlying myocardial perfusion abnormalities in that particular group of patients have been studied extensively and 
FIGURE 2. Percentage of patients with abnormal myocardial perfusion per FMD quartile or CAC score category. Prevalence of abnormal myocardial perfusion increased with decreasing FMD (A), whereas no clear trend was observed among increasing CAC score categories (B).
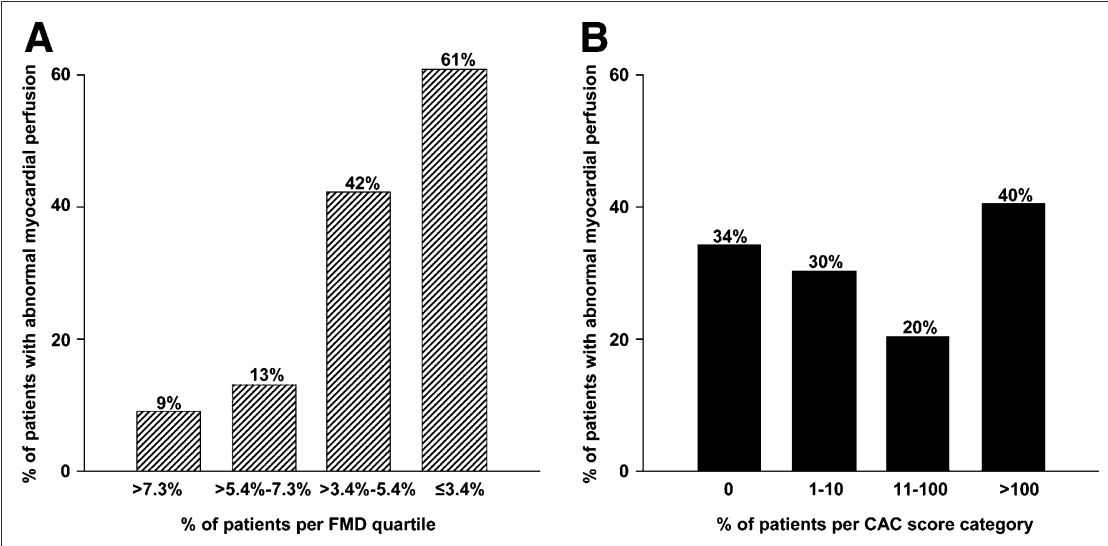

revealed the presence of diffuse coronary atherosclerosis and endothelial dysfunction as potential causal factors (46). However, little is known about the contribution of these parameters to the occurrence of myocardial perfusion defects in asymptomatic patients with diabetes mellitus. Therefore, in the current study we examined the influence of endothelial dysfunction and nonobstructive atherosclerotic plaque burden on myocardial perfusion in asymptomatic diabetic patients without epicardial obstructive CAD.

\section{Assessment of Endothelial Function by FMD}

Assessment of systemic endothelial function by FMD has been shown to relate closely to coronary vasoreactivity
$(8,9)$. To date, measurement of brachial FMD is accepted as the most validated and reproducible noninvasive technique for assessment of endothelial function (7). Interobserver reproducibility of the baseline and maximum postischemic brachial artery is satisfactory, with diameter variations of approximately $4 \%$, and has improved with the introduction of semiautomated wall-track systems $(10,18)$. In contrast, the intraobserver reproducibility is limited by within-subject variability and surrounding factors, as vasoreactivity fluctuates through the day and is further influenced by stress, temperature, diet, and glucose levels (10). Therefore, FMD appears to be less applicable to individual serial testing. However, a panel of experts has recognized the test as

\section{TABLE 2. Predictors of Extent of Abnormal Myocardial Perfusion (SSS) in Patients Without Obstructive}

Epicardial CAD

\begin{tabular}{|c|c|c|c|c|}
\hline \multirow[b]{2}{*}{ Predictor } & \multicolumn{2}{|c|}{ Univariate analysis } & \multicolumn{2}{|c|}{ Multivariate analysis } \\
\hline & $\operatorname{Exp} \beta(95 \% \mathrm{Cl})$ & $P$ & $\operatorname{Exp} \beta(95 \% \mathrm{Cl})$ & $P$ \\
\hline FMD (\%) & $0.61(0.48-0.78)$ & $<0.001$ & $0.58(0.43-0.75)$ & $<0.001$ \\
\hline CAC score & $1.00(0.99-1.00)$ & 0.22 & - & - \\
\hline Age (y) & $1.01(0.98-1.06)$ & 0.45 & - & - \\
\hline Male sex & $1.57(0.66-3.75)$ & 0.31 & - & - \\
\hline Diabetes mellitus duration (y) & $0.98(0.95-1.01)$ & 0.33 & - & - \\
\hline Body mass index $\left(\mathrm{kg} / \mathrm{m}^{2}\right)$ & $1.02(0.95-1.09)$ & 0.59 & - & - \\
\hline Hemoglobin A1c (mmol/L) & $0.76(0.55-1.04)$ & 0.09 & $0.50(0.31-0.82)$ & 0.01 \\
\hline Smoking & $0.91(0.33-2.53)$ & 0.86 & - & - \\
\hline Family history of CAD & $0.93(0.39-2.21)$ & 0.86 & $0.36(0.11-1.19)$ & 0.09 \\
\hline Hypercholesterolemia & $1.65(0.64-4.29)$ & 0.30 & $4.04(1.07-15.24)$ & 0.04 \\
\hline Hypertension & $1.22(0.51-2.90)$ & 0.66 & - & - \\
\hline Type 1 diabetes mellitus & $0.16(-0.90-1.22)$ & 0.77 & - & - \\
\hline Treatment with only diet & $1.57(-0.78-3.91)$ & 0.19 & - & - \\
\hline Treatment with oral agents & $-0.41(-1.51-0.68)$ & 0.45 & - & - \\
\hline Treatment with insulin & $0.15(-1.18-1.47)$ & 0.83 & - & - \\
\hline Statin use & $0.36(-0.71-1.43)$ & 0.50 & - & - \\
\hline Aspirin use & $0.79(-0.70-2.27)$ & 0.30 & - & - \\
\hline $\begin{array}{l}\text { Angiotensin-converting } \\
\text { enzyme inhibitor use }\end{array}$ & $-0.14(-1.59-1.31)$ & 0.85 & - & - \\
\hline$\beta$-blocker use & $0.15(-1.76-2.05)$ & 0.88 & - & - \\
\hline Angiotensin II antagonist use & $-0.99(-2.57-0.58)$ & 0.21 & - & - \\
\hline Calcium channel blocker use & $0.95(-1.06-2.97)$ & 0.35 & - & - \\
\hline Diureticum use & $0.16(-1.52-1.85)$ & 0.85 & - & - \\
\hline
\end{tabular}


appropriate for cohort studies, provided that patient and surrounding factors are standardized (19). FMD ranges from approximately $20 \%$ in young adults to $0 \%$ in patients with established CAD (20). In diabetic patients, mean FMD values tend to be lower as well (range, $0 \%-12 \%$ ) (20). In diabetes mellitus, endothelial dysfunction is suspected to be induced by hyperglycemia (sorbitol, hexosamine, protein kinase $\mathrm{C}$, and advanced glycation end product pathways) and insulin resistance, which result in mitochondrial superoxide overproduction. As a consequence, nitric oxide availability is negatively affected, leading to endothelial dysfunction $(21,22)$.

\section{Relationship with Myocardial Perfusion}

In the current study, FMD of the brachial artery was further decreased in diabetic patients with abnormal myocardial perfusion. Several studies have described an association between endothelial dysfunction and the occurrence of myocardial ischemia due to impaired endotheliumdependent coronary vasodilation during stress (23). It is therefore presumable that in the current study insufficient vasomotor response in the coronary microvasculature due to endothelial dysfunction may indeed have resulted in relative hypoperfusion during stress (reflected by reversible perfusion abnormalities on SPECT) in the absence of obstructive epicardial CAD (23). However, endothelial dysfunction has also been shown to affect resting myocardial perfusion (24). Accordingly, hypoperfusion of the microvasculature during rest may occur. Interestingly, in our study most fixed perfusion abnormalities comprised a mild decrease in tracer uptake, which has been linked to hypoperfused but viable myocardial tissue rather than scarring (25). Nevertheless, impaired coronary endothelial function has also been suggested as a cause of myocardial infarction in patients with minimally obstructive disease, possibly through prolonged vasoconstriction (26). Therefore, it is possible that the fixed abnormalities comprising severely decreased tracer uptake, which we observed in 2 patients, may indeed reflect loss of viable myocardium. Finally, the possibility remains that some of the fixed perfusion abnormalities were due to attenuation. However, in the current study, regional wall motion on gated SPECT images was analyzed for optimal differentiation between true perfusion abnormalities and attenuation artifacts.

Importantly, FMD was shown to be an independent predictor of the extent of abnormal perfusion, even after correction for occult coronary atherosclerosis by means of CAC scores. Accordingly, it appears that in the absence of epicardial obstructive CAD, endothelial dysfunction may have a greater impact on myocardial perfusion than the extent of diffuse atherosclerosis. Further investigations are needed to confirm these observations.

\section{Potential Clinical Implications}

The direct cardiovascular prognostic consequence of endothelial dysfunction in diabetic patients remains to be determined. In previous studies of nondiabetic patients, impaired endothelial function has been shown to predict cardiovascular events (27). In addition, Bugiardini et al. showed that reduced coronary vasoreactivity in angiographically normal coronaries was a predecessor of overt atherosclerosis on angiography after a long-term follow-up of $10 \mathrm{y}$ (28). Therefore, dysfunction of the endothelium seems to occur in early stages of vascular disease and may be reversible. Accordingly, cholesterol-lowering therapy has been shown to improve endothelium-dependent vasomotion in patients with hypercholesterolemia, as well as myocardial perfusion in a nondiabetic cohort of patients with previous evidence of myocardial perfusion defects on SPECT $(29,30)$. In line with these observations, inducible ischemia was shown to resolve in $79 \%$ of asymptomatic diabetic patients who had abnormal myocardial perfusion at baseline in the Detection of Ischemia in Asymptomatic Diabetics study (31). This recovery was associated with intensification of treatment with statins, aspirin, and angiotensin-converting enzyme inhibitors.

Thus, in the current study, abnormal myocardial perfusion not attributable to obstructive CAD was related to impaired endothelial function. Because this condition is most likely to be an early stage of vascular disease amenable to treatment, intensification of antiatherogenic therapy seems to be indicated in asymptomatic diabetic patients with perfusion abnormalities even in absence of obstructive epicardial CAD.

Furthermore, considering that in diabetes mellitus abnormal myocardial perfusion is often observed in the absence of epicardial obstructive $\mathrm{CAD}$, the question emerges of whether this test should be accompanied by a noninvasive anatomic test such as multislice CT coronary angiography. Indeed, referral of all asymptomatic diabetic patients with abnormal myocardial perfusion for invasive coronary angiography should be avoided considering that many patients will not have obstructive epicardial stenosis amenable to revascularization. Accordingly, combined noninvasive imaging of myocardial perfusion imaging by SPECT and coronary anatomy by multislice CT may allow a more comprehensive and accurate assessment of $\mathrm{CAD}$ and facilitate further management. However, routine combination of these 2 noninvasive imaging modalities carries the disadvantage of increased costs and radiation exposure. Possibly, multislice CT coronary angiography could have the greatest incremental value in asymptomatic diabetic patients with abnormal myocardial perfusion, to differentiate between obstructive epicardial CAD and endothelial dysfunction as a causal factor.

\section{Study Limitations}

First, the size of the study population was limited. In addition, we acknowledge that brachial FMD is a measure of systemic endothelial function and not a direct measure of coronary endothelial function. However, previous studies have shown a good correlation between brachial FMD and direct invasive measures of coronary endothelial function $(13,14)$. Furthermore, the radiation burden associated with 
combined multislice CT (64-slice multislice CT $[\sim 9-15$ $\mathrm{mSv}]$ ) and SPECT myocardial perfusion imaging $\left({ }^{99 \mathrm{~m} T c}\right.$ sestamibi $[\sim 7 \mathrm{mSv}$ per scan]) is a limitation $(32,33)$. However, the radiation dose of multislice CT has been decreased significantly with the dedicated dose-reduction multislice CT acquisition techniques that have recently become available $(34,35)$. Finally, no follow-up data were available, and whether our observations have prognostic implications should be evaluated in prospective follow-up studies.

\section{CONCLUSION}

In asymptomatic diabetic patients with no obstructive epicardial CAD, abnormal myocardial perfusion is common and is strongly associated with impaired endothelial function. In view of the likely reversible nature of endothelial dysfunction and the cardiovascular prognostic value of abnormal myocardial perfusion in asymptomatic diabetic patients, it is presumable that asymptomatic diabetic patients with abnormal myocardial perfusion will benefit from intensified pharmacologic treatment even in the absence of obstructive epicardial CAD.

\section{ACKNOWLEDGMENTS}

The study was supported in part by grants from Medtronic, Biotronik, Boston Scientific, BMS Medical Imaging, St. Jude Medical, GE Healthcare, Edwards Lifesciences, Astra Zeneca, Pfizer, and MSD.

\section{REFERENCES}

1. Consensus development conference on the diagnosis of coronary heart disease in people with diabetes: 10-11 February 1998, Miami, Florida. American Diabetes Association. Diabetes Care. 1998;21:1551-1559.

2. Scholte AJ, Schuijf JD, Kharagitsingh AV, et al. Different manifestations of coronary artery disease by stress SPECT myocardial perfusion imaging, coronary calcium scoring, and multislice CT coronary angiography in asymptomatic patients with type 2 diabetes mellitus. $J$ Nucl Cardiol. 2008;15:503-509.

3. Kang X, Berman DS, Lewin H, et al. Comparative ability of myocardial perfusion single-photon emission computed tomography to detect coronary artery disease in patients with and without diabetes mellitus. Am Heart J. 1999;137:949-957.

4. Bellamy MF, Goodfellow J, Tweddel AC, Dunstan FD, Lewis MJ, Henderson AH. Syndrome X and endothelial dysfunction. Cardiovasc Res. 1998;40:410-417.

5. Masci PG, Laclaustra M, Lara JG, Kaski JC. Brachial artery flow-mediated dilation and myocardial perfusion in patients with cardiac syndrome X. Am J Cardiol. 2005;95:1478-1480.

6. Rodés-Cabau J, Candell-Riera J, Angel J, et al. Relation of myocardial perfusion defects and nonsignificant coronary lesions by angiography with insights from intravascular ultrasound and coronary pressure measurements. Am J Cardiol. 2005;96:1621-1626.

7. Deanfield JE, Halcox JP, Rabelink TJ. Endothelial function and dysfunction: testing and clinical relevance. Circulation. 2007;115:1285-1295.

8. Anderson TJ, Uehata A, Gerhard MD, et al. Close relation of endothelial function in the human coronary and peripheral circulations. J Am Coll Cardiol. 1995;26:1235-1241.

9. Pellegrino T, Storto G, Filardi PP, et al. Relationship between brachial artery flow-mediated dilation and coronary flow reserve in patients with peripheral artery disease. J Nucl Med. 2005;46:1997-2002.

10. Hijmering ML, Stroes ES, Pasterkamp G, Sierevogel M, Banga JD, Rabelink TJ. Variability of flow mediated dilation: consequences for clinical application. Atherosclerosis. 2001;157:369-373.

11. Hansen CL, Goldstein RA, Akinboboye OO, et al. American Society of Nuclear Cardiology. Myocardial perfusion and function: single photon emission computed tomography. J Nucl Cardiol. 2007;14:e39-e60.
12. Hoeks AP, Willekes C, Boutouyrie P, Brands PJ, Willigers JM, Reneman RS. Automated detection of local artery wall thickness based on M-line signal processing. Ultrasound Med Biol. 1997;23:1017-1023.

13. Exercise perfusion imaging in coronary artery disease: physiology and diagnosis. In: Iskandrian AS, Verani MS. Nuclear Cardiac Imaging: Principles and Applications. Philadelphia, PA: FA Davis; 1996:73-143.

14. Thomas GS, Miyamoto MI, Morello AP III, et al. Technetium 99m sestamibi myocardial perfusion imaging predicts clinical outcome in the community outpatient setting. The Nuclear Utility in the Community (NUC) Study. J Am Coll Cardiol. 2004;43:213-223.

15. Zellweger MJ, Hachamovitch R, Kang X, et al. Prognostic relevance of symptoms versus objective evidence of coronary artery disease in diabetic patients. Eur Heart J. 2004;25:543-550.

16. Valensi P, Pariès $\mathrm{J}$, Brulport-Cerisier $\mathrm{V}$, et al. Predictive value of silent myocardial ischemia for cardiac events in diabetic patients: influence of age in a French multicenter study. Diabetes Care. 2005;28:2722-2727.

17. Elhendy A, Huurman A, Schinkel AF, et al. Association of ischemia on stress ${ }^{99 \mathrm{~m}}$ Tc-tetrofosmin myocardial perfusion imaging with all-cause mortality in patients with diabetes mellitus. J Nucl Med. 2005;46:1589-1595.

18. De Roos NM, Bots ML, Schouten EG, Katan MB. Within-subject variability of flow-mediated vasodilation of the brachial artery in healthy men and women: implications for experimental studies. Ultrasound Med Biol. 2003;29:401-406.

19. Corretti MC, Anderson TJ, Benjamin EJ, et al. International Brachial Artery Reactivity Task Force. Guidelines for the ultrasound assessment of endothelialdependent flow-mediated vasodilation of the brachial artery: a report of the International Brachial Artery Reactivity Task Force. J Am Coll Cardiol. 2002;39:257-265.

20. Bots ML, Westerink J, Rabelink TJ, de Koning EJ. Assessment of flow-mediated vasodilatation (FMD) of the brachial artery: effects of technical aspects of the FMD measurement on the FMD response. Eur Heart J. 2005;26:363-368.

21. Creager MA, Lüscher TF, Cosentino F, Beckman JA. Diabetes and vascular disease: pathophysiology, clinical consequences, and medical therapy-part I. Circulation. 2003;108:1527-1532.

22. Tan KC, Chow WS, Ai VH, Metz C, Bucala R, Lam KS. Advanced glycation end products and endothelial dysfunction in type 2 diabetes. Diabetes Care. 2002;25:1055-1059.

23. Hasdai D, Gibbons RJ, Holmes DR Jr, Higano ST, Lerman A. Coronary endothelial dysfunction in humans is associated with myocardial perfusion defects. Circulation. 1997;96:3390-3395.

24. Johnson NP, Gould KL. Clinical evaluation of a new concept: resting myocardial perfusion heterogeneity quantified by Markovian analysis of PET identifies coronary microvascular dysfunction and early atherosclerosis in 1,034 subjects. J Nucl Med. 2005;46:1427-1437.

25. Dakik HA, Howell JF, Lawrie GM, et al. Assessment of myocardial viability with ${ }^{99 m} \mathrm{Tc}$-sestamibi tomography before coronary bypass graft surgery: correlation with histopathology and postoperative improvement in cardiac function. Circulation. 1997;96:2892-2898.

26. Lerman A, Zeiher AM. Endothelial function: cardiac events. Circulation. 2005; 111:363-368.

27. Gokce N, Keaney JF Jr, Hunter LM, et al. Predictive value of noninvasively determined endothelial dysfunction for long-term cardiovascular events in patients with peripheral vascular disease. J Am Coll Cardiol. 2003;41:1769-1775.

28. Bugiardini R, Manfrini O, Pizzi C, Fontana F, Morgagni G. Endothelial function predicts future development of coronary artery disease: a study of women with chest pain and normal coronary angiograms. Circulation. 2004;109:2518-2523.

29. Egashira K, Hirooka Y, Kai H, et al. Reduction in serum cholesterol with pravastatin improves endothelium-dependent coronary vasomotion in patients with hypercholesterolemia. Circulation. 1994;89:2519-2524.

30. Mostaza JM, Gomez MV, Gallardo F, et al. Cholesterol reduction improves myocardial perfusion abnormalities in patients with coronary artery disease and average cholesterol levels. J Am Coll Cardiol. 2000;35:76-82.

31. Wackers FJ, Chyun DA, Young LH, et al. Detection of Ischemia in Asymptomatic Diabetics (DIAD) investigators. Resolution of asymptomatic myocardial ischemia in patients with type 2 diabetes in the Detection of Ischemia in Asymptomatic Diabetics (DIAD) study. Diabetes Care. 2007;30:2892-2898.

32. Einstein AJ, Moser KW, Thompson RC, Cerqueira MD, Henzlova MJ. Radiation dose to patients from cardiac diagnostic imaging. Circulation. 2007;116:1290-1305.

33. Einstein AJ. Heart. 2008;94:1519-1521.

34. Husmann L, Valenta I, Gaemperli O, et al. Feasibility of low-dose coronary CT angiography: first experience with prospective ECG-gating. Eur Heart J. 2008;29:191-197.

35. Rybicki FJ, Otero HJ, Steigner ML, et al. Initial evaluation of coronary images from 320-detector row computed tomography. Int J Cardiovasc Imaging. 2008; 24:535-546. 